\title{
The cusp: a window for particle exchange between the radiation belt and the solar wind
}

\author{
X.-Z. Zhou ${ }^{1,2}$, T. A. Fritz ${ }^{3}$, Q.-G. Zong ${ }^{4,2}$, Z. Y. Pu ${ }^{1}$, Y.-Q. Hao ${ }^{1}$, and J.-B. Cao ${ }^{2}$ \\ ${ }^{1}$ Institute of Space Physics and Applied Technology, Peking University, Beijing 100871, China \\ ${ }^{2}$ Key Laboratory for Space Weather, Chinese Academy of Sciences, Beijing, China \\ ${ }^{3}$ Center for Space Physics, Boston University, Boston, MA 02215, USA \\ ${ }^{4}$ Center for Atmospheric Research, University of Massachusetts, Lowell, MA 01854, USA
}

Received: 18 February 2006 - Revised: 28 August 2006 - Accepted: 13 October 2006 - Published: 22 November 2006

\begin{abstract}
The study focuses on a single particle dynamics in the cusp region. The topology of the cusp region in terms of magnetic field iso-B contours has been studied using the Tsyganenko 96 model (T96) as an example, to show the importance of an off-equatorial minimum on particle trapping. We carry out test particle simulations to demonstrate the bounce and drift motion. The "cusp trapping limit" concept is introduced to reflect the particle motion in the high latitude magnetospheric region. The spatial distribution of the "cusp trapping limit" shows that only those particles with near $90^{\circ}$ pitch-angles can be trapped and drift around the cusp. Those with smaller pitch angles may be partly trapped in the iso-B contours, however, they will eventually escape along one of the magnetic field lines. There exist both open field lines and closed ones within the same drift orbit, indicating two possible destinations of these particles: those particles being lost along open field lines will be connected to the surface of the magnetopause and the solar wind, while those along closed ones will enter the equatorial radiation belt. Thus, it is believed that the cusp region can provide a window for particle exchange between these two regions. Some of the factors, such as dipole tilt angle, magnetospheric convection, IMF and the Birkeland current system, may influence the cusp's trapping capability and therefore affect the particle exchanging mechanism. Their roles are examined by both the analysis of cusp magnetic topology and test particle simulations.
\end{abstract}

Keywords. Magnetospheric physics (Energetic particles, trapped; Magnetopause, cusp and boundary layers; Solar wind-magnetosphere interactions)

Correspondence to: X.-Z. Zhou

(zhouxz@pku.edu.cn)

\section{Introduction}

The polar cusp, as an essential part of the Earth's highlatitude dayside magnetosphere, is a region with magnetosheath plasma and electromagnetic turbulence (e.g. Cargill et al., 2005). The properties of the cusp region (location, extent, etc) are highly influenced by the IMF and the dipole tilt angle (Tsyganenko and Russell, 1999; Zhou et al., 2000; Merka et al., 2002).

The cusp is also observed to contain an off-equatorial magnetic minimum region (e.g. Newell and Meng, 1987; Zhou et al., 1997). Therefore, energetic particles in the cusp region are affected by a mirror force that always points to the magnetic minimum, so it is natural to predict a cusp trapping region for energetic particles with near $90^{\circ}$ pitch angles. Based on the Tsyganenko 96 (hereinafter as T96) model (Tsyganenko and Stern, 1996), test particle simulations were made to point out that a particle can drift on a closed path around the outer cusp (Sheldon et al., 1998). These simulations further demonstrated that the trapping time would be infinite if no temporal variations were considered. The concept of cusp trapping was supported by Kirpichev et al. (1999) who examined the spatial distribution of energetic particles in the outer cusp using INTERBALL-1 data. Pugacheva et al. $(2004,2005)$ further suggested the existence of a "cusp radiation belt" containing energetic particles and elaborately discussed how its occurrence and location are affected by the level of geomagnetic activity and dipole tilt angle.

In order to explain the origin of these particles in the cusp region, Delcourt and Sauvaud $(1998,1999)$ followed the idea of Antonova and Shabansky (1968) and Shabansky (1971) and suggested that these particles could originated at the equatorial plane, based upon the existence of an offequatorial minimum in the frontside sector. Thus, when the plasma sheet particles initially mirroring near the equatorial

Published by Copernicus GmbH on behalf of the European Geosciences Union. 


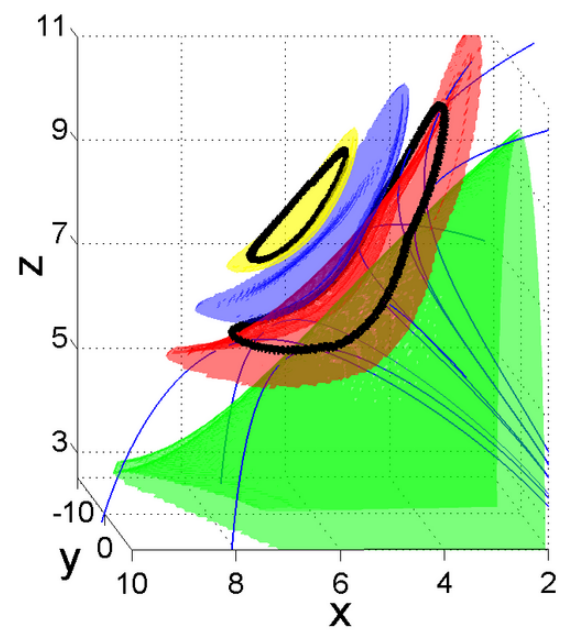

Fig. 1. T96 magnetic field topology in the cusp region during spring equinox (IMF: $0 \mathrm{nT} B_{y}$ and $-1 \mathrm{nT} B_{z} ; D_{s t}$ : -22.0 ; Solar Wind Pressure: $2.53 \mathrm{nPa}$ ). Blue lines are field lines. Particles will drift around the cusp region as closed orbits, two examples of which are shown here as black lines. The yellow, blue, red and green planes are four contour planes, representing $20 \mathrm{nT}, 30 \mathrm{nT}, 40 \mathrm{nT}$ and $60 \mathrm{nT}$, respectively.

plane drift sunward, they will move away from the equator to the cusp minimum under the effect of cuspward mirror force.

However, it should be kept in mind that the T96 model is not a perfect model, especially in the cusp region. A statistical survey of Cluster data was made by Lavraud et al. (2004) to represent the magnetic field deviations between observations and the T96 model. The observed magnetic field strength was shown to be even weaker than the prediction of T96, probably caused by an additional diamagnetic current systems. Although there was an attempt by Tsyganenko and Russell (1999) to incorporate the effect of a diamagnetic depression in the T96 model, the modified model still had some difficulties in data fitting and thus could not represent the real magnetic field in the cusp region.

In this paper, we suggest the term of "cusp trapping limit" (CTL) of energetic particles after analyzing the magnetic topology of the cusp region using the T96 model. Only those particles with larger pitch angles than the CTL can be locally trapped, while the others will get lost during their bounce motion along the field lines. The value of CTL is shown to be spatially dependent, indicating regions for which it is more probable for particles to escape as they drift. Moreover, due to the sensitivity of the cusp on external conditions (IMF, dipole tilt angle, etc.), the value of CTL also varies with time, further activating the particle escaping mechanism. Because of the coexistence of closed field lines and open ones in the cusp region, shown in T96 and also in observations (e.g. Fuselier et al., 1995; Zhou et al., 2000), these particles are connected to both the traditional dipole trapping region and the solar wind. Test particle simulations are carried out and described below which confirm the couplings between these regions.

It should be noted again that T96 is not an ideal model for the study of cusp, and this does reduce the accuracy of the quantitive results. The analysis methods and test particle simulations should be applied to more accurate models in the future when these models are available.

\section{Magnetic topology in the cusp region}

In order to better understand the relationship between magnetic topology (magnetic minimum in the cusp region) and the motion of energetic particles, as an example, Fig. 1 visualizes some of the cusp magnetic contour planes during spring equinox, based on T96 model.

In Fig. 1, four contour planes are presented, and each of them has a certain value of $|B|(20 \mathrm{nT}, 30 \mathrm{nT}, 40 \mathrm{nT}$ and $60 \mathrm{nT}$ for yellow, blue, red and green, respectively). As can be seen clearly in this figure, the magnetic field strength is relatively small in the cusp region, so if a test particle is launched at a magnetic field minimum with a $90^{\circ}$ pitch angle, admittedly without considering the effect of electric fields, the mirror force will always keep the particle at the magnetic minimum points of different field lines, and the particle will drift around the cusp, due to the existence of magnetic gradients (Sheldon et al., 1998). The two black lines in Fig. 1 represent two test particles following this type of orbit in a steady T96 cusp, with different initial positions. Each of these particles is trapped by a set of field minima with a magnetic magnitude of around $20 \mathrm{nT}$ and $40 \mathrm{nT}$, respectively.

Based on the fact that each field line approaching the cusp region has a magnetic minimum there, we can compose all the magnetic minimum points along their field lines to form a "cusp B-field minimum plane", which can be directly compared with the dipole equatorial plane. The two test particles described above are found to be well trapped on the plane (see Fig. 2a), suggesting two "cusp-shells" analogous to L-shells of the dipole field. Their drift paths are basically close, implying the adiabatic motions of these particles, and also suggesting the existence of a stably-trapped region in the cusp. This is similar to the pseudo-trapped drift shells in the equatorial plane, which can only trap particles for some time before they meet with the magnetopause. There also exists a pseudo-trapped region in the "cusp minimum plane". Particles originally trapped there will not complete their drift circuits in the cusp region, but they will instead drift for a while, find themselves on a field line without a cusp minimum and escape along the field. The boundary between the stablytrapped region and the pseudo-trapped region might be the outer edge of the spatial "cusp radiation belt" extent, analogous to the outer edge of the radiation belts. Figure 2a shows the shape of the stably-trapped region of the "cusp minimum plane", with the color representing the magnetic strength of 

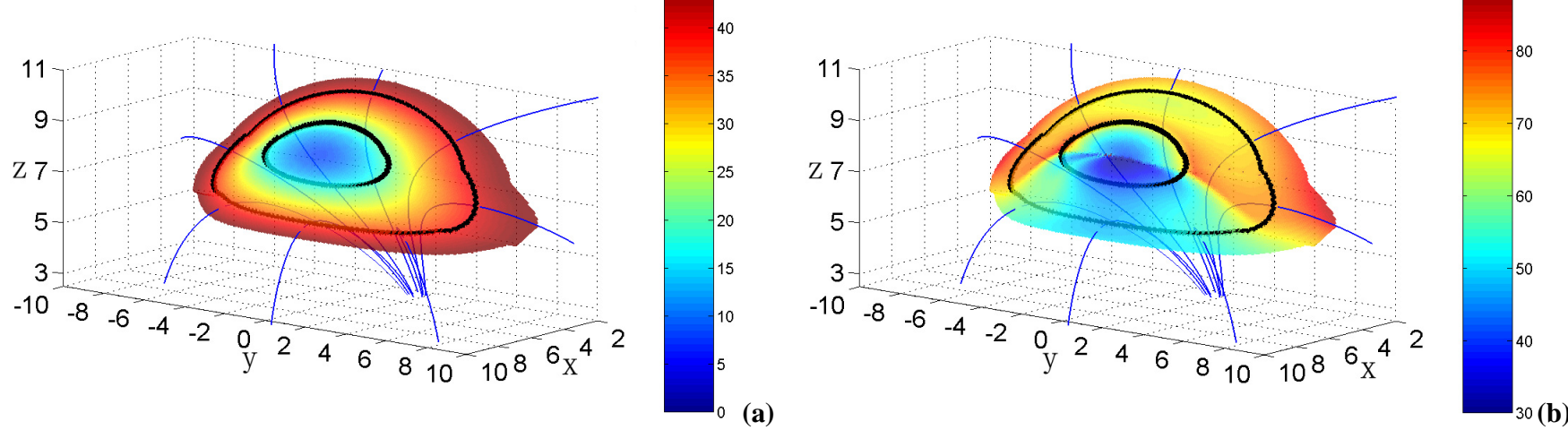

Fig. 2. The shape of the "cusp B-field minimum plane" during spring equinox (IMF: $0 \mathrm{nT} B_{y}$ and $-1 \mathrm{nT} B_{z} ; D_{s t}$ : -22.0 ; Solar (a) The color represents the magnetic strength of each field minimum point in nT. (b) The color represents the pitch angle at minimum point of a given field line associated with the "cusp trapping limit".
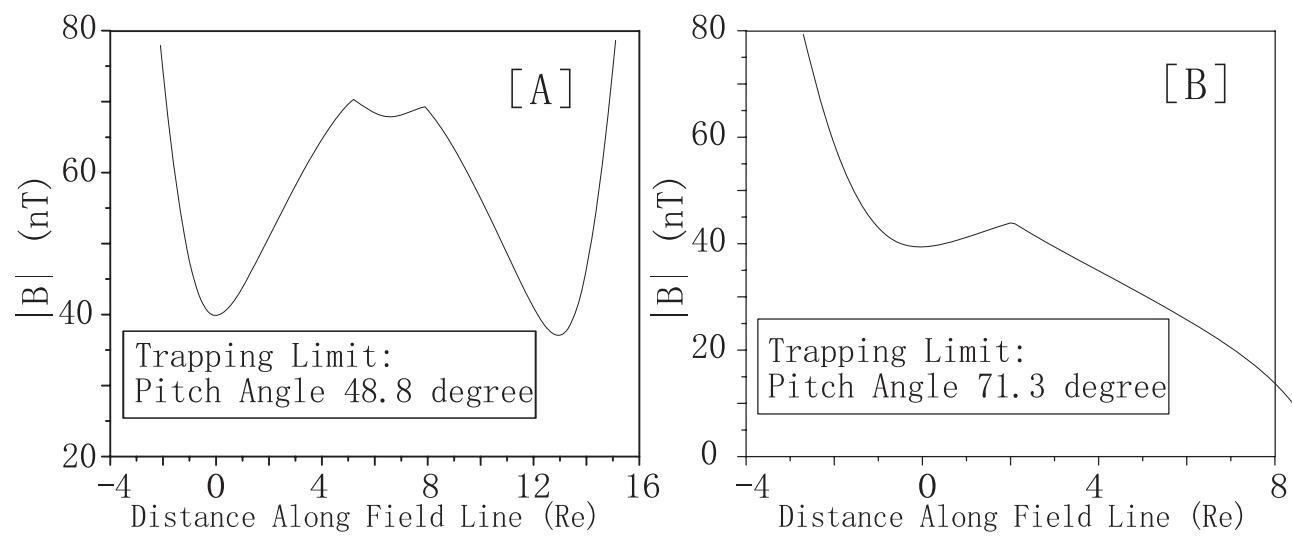

Fig. 3. Two examples of magnetic strength as a function of the distance along a field line in T96 model. The $\mathrm{X}=0$ point in either example represents a magnetic minimum where the test particle ever passed by in the northern cusp. (A) The example when the particle is in a minimum of closed field lines. The two minimum regions represent northern cusp and southern cusp, respectively. (B) The example of open ones with only one minimum along the field line.

each field minimum point, i.e. different colors represent different "cusp-shells".

Before discussing the external conditions that can modify the shape of the stably-trapped region of the cusp, let us focus on the trajectory of the test particle trapped within the $40 \mathrm{nT}$ contour plane in more detail, as an example. In this tracing calculation, the full particle dynamics are considered by applying a fourth-order Runge-Kutta method with an automatic correction for energy conservation in each step. A $50 \mathrm{keV}$ proton is launched at the minimum of a closed field line near the dayside cusp, drifting to the west towards the flank region. After that, it begins a drift upward to larger values of GSM Z, staying in the minima of open field lines. Then it moves toward the dusk flank and returns back to the closed field line region. The distinct configurations of both closed field line regions and open ones within the same orbit are displayed in Fig. 3, presenting $|B|$ as the function of the distance along certain field lines.

If we treat these two kinds of configurations as two magnetic mirrors, each of which has a magnetic minimum and a maximum in the northern cusp, the "cusp trapping limit" (CTL) can be accordingly defined as the loss cone pitch angle of those magnetic mirrors, say, $\arcsin \left(B_{\min } / B_{\max }\right)^{1 / 2}$. They are shown to be different, indicating a relatively higher trapping efficiency in the geometry of closed field lines because of its lower CTL value. Given a certain magnetic model, the values of CTL along entire "cusp minimum plane" can be calculated to illustrate the spatial distribution of trapping areas: Energetic particles can be better trapped in the regions of low CTL values, while the places with relatively larger values of CTL correspond to the active regions of particle escaping from the cusp region. 


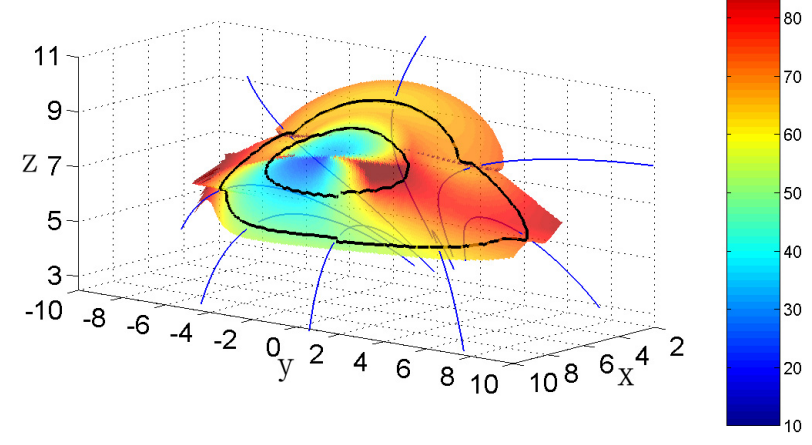

Fig. 4. Similar to Fig. $2 b$, but with a negative IMF $B_{y}$ of $-5 \mathrm{nT}$. The color shows the spatial distribution of the "cusp trapping limit" in T96 model.

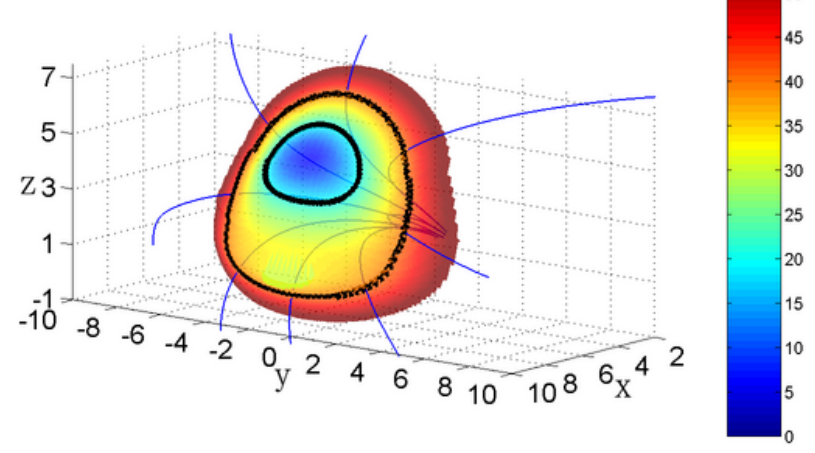

Fig. 5. The shape of the "cusp minimum plane" during summer solstice, analogous to Fig. 2a.

The distribution example for the T96 model during spring equinox is displayed in Fig. 2b. It can be clearly seen that there are two peaks of the CTL on the duskside and dawnside regions of the cusp, suggesting a relatively weak capability of particle trapping in these regions. Along the drift paths of energetic particles (clockwise for positive particles and anti-clockwise for electrons), the regions with increasing CTL values will correspond to the regions where particles can more readily escape. On the contrary, in the regions with decreasing CTL values, there will be particles captured from other regions which become trapped in the cusp, as the orbits calculated by Delcourt and Sauvaud (1999) demostrated. Based on the opposite drift direction of positive and negative particles, it is clear that the regions with a strong tendency for positive particles to escape from the cusp (higher latitude duskside cusp and lower latitude dawnside cusp in this case) are actually regions that will feed electrons into the cusp, and vice versa.

In this case, all of these regions are situated on closed field lines. Particles escaping from these regions will move along the field lines to find their mirror points in the Southern
Hemisphere (see Fig. 3a), bounce back and remain trapped in a relatively larger scale, i.e. the traditional trapping region. Similarly, those particles becoming trapped from these regions are captured from the traditional trapping region, suggesting a mechanism to exchange particles between the cusp region and the radiation belt.

The features will be slightly different if the effect of IMF $B_{y}$ is considered. By studying POLAR data, Zhou et al. (2000) suggested that the cusp would move dawnward with open field lines in the duskside sector during negative IMF, and vice versa. Actually, the T96 cusp also holds these properties. Figure 4 shows the T96 spatial distribution of "cusp trapping limit" when IMF $B_{y}$ equals to $-5 \mathrm{nT}$. There still exist two peaks in the duskside and dawnside sector of the cusp region, however, the field lines in the dawnside peak are still closed while those in the duskside peak are open, in agreement with the observation. In this case, particles that either move away from the cusp region or move into the cusp through the dawnside peak will still be connected to the radiation belt, while those through the duskside peak will get lost into or are coming from the solar wind (see Fig. 3b). On the contrary, if the IMF $B_{y}$ is positive ( $5 \mathrm{nT}$ ), the duskside peak will be closed while the dawnside peak would be open. Because particles in different sections of the cusp, which can be easily connected to either the equator or the solar wind, are topologically linked together via drift motions, it is believed that the cusp can play a role as a bridge between the solar wind and the radiation belt for energetic particles.

The effect of the dipole tilt angle on the cusp trapping region can also be evaluated based on the T96 model. Figure 5 shows the shape of the "cusp minimum plane" during summer solstice. During winter solstice, the "cusp minimum plane" in the Northern Hemisphere disappears, which is consistent with the conclusion of Pugacheva et al. (2005). We will examine the reason for the existence and extent of the "cusp radiation belt" dependence on the geomagnetic dipole tilt. For example, during winter solstice, with relatively larger tilt angles, the northern cusp region should shift antisunward, with relatively less impact there by the solar wind. Therefore, the magnetic field would approximate the undisturbed dipole field, with no magnetic minimum in the northern cusp. As a consequence, the cusp region would not trap particles, and accordingly lose its ability of providing any window for particle exchange with other regions.

\section{Test particle simulations}

The features discussed above on the T96 cusp magnetic topology can be used to predict the factors which can affect the motion of particles in the cusp region. Test particle simulations are performed to test the validity of their effects. For simplicity, these factors are examined separately in a steady-state T96 cusp region, with the other factors kept fixed. 

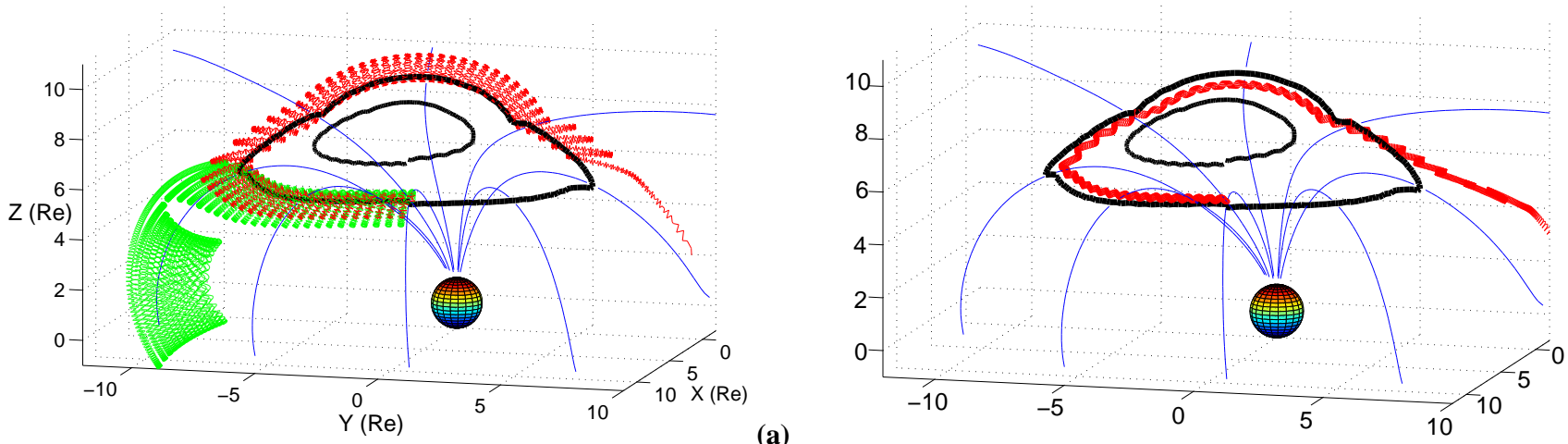

(a)

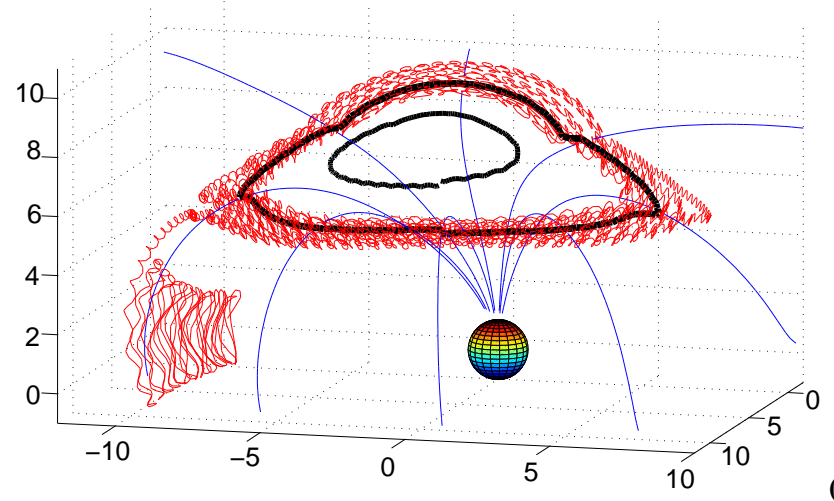

(c)

Fig. 6. Trajectory of several test particles during spring equinox (IMF: $-5 \mathrm{nT} B_{y}$ and $-1 \mathrm{nT} B_{z} ; D_{s t}$ : -22.0 ; Solar Wind Pressure: $2.53 \mathrm{nPa}$ ). The black lines shows two $90^{\circ} 50 \mathrm{keV}$ protons drifting around the cusp region. (a) The green line represents the trajectory of another $50 \mathrm{keV}$ proton initially with a $70^{\circ}$ pitch angle, while the red line shows the same proton with a pitch angle of $75^{\circ}$. No electric field is considered here. (b) The effect of convection electric field is studied, and the red line represents a $50 \mathrm{keV}$ proton's orbit initially with a $90^{\circ}$ pitch angle, escaping in the duskside cusp. (c) The orbit of a $100 \mathrm{keV}$ proton with an initially $90^{\circ}$ pitch angle. It drifts around the cusp for six times and escape to the equatorial plane via the dawnside cusp. Again, no electric field is considered. The particle's nonadiabatic behavior is considered to be caused by the T96 Birkeland current term.

Tilt angle: According to the discussions, the existence of a "cusp minimum plane" strongly depends on the geomagnetic dipole tilt. Test particle simulations are performed during spring equinox, summer solstice and winter solstice. Being identical with the prediction, the $90^{\circ}$ test particle (50 keV proton) can drift around the northern cusp region during both spring equinox (see the outer black circle in Fig. 2a) and summer solstice (the outer black circle in Fig. 5), while at winter solstice it can not be trapped. Instead, the particle simply bounces away from the northern cusp region (not shown here).

IMF $B_{y}:$ In the case of nonzero- $B_{y}$, one of the two peaks is no longer situated on closed field lines. For example, when the IMF $B_{y}$ equals to $-5 \mathrm{nT}$, the magnetic field lines in the duskside peak are open, while those in the dawnside cusp are still closed. As was predicted before, duskside detrapping of particles will occur on open field lines and they will disappear into the solar wind, while those particles escaping in the dawnside sector will be trapped on a relatively larger scale, such as being transferred to the radiation belt and drifting around the Earth.

Test particle tracing calculations are made to prove the result, shown in Fig. 6a. The red line displays the trajectory of a $50 \mathrm{keV}$ proton escaping the cusp region in the duskside sector while the green one corresponds to another $50 \mathrm{keV}$ proton escaping in the dawnside sector. Their distinct orbits, one getting lost to the solar wind while the other one merging into the dipole trapping region, are in agreement with the different topologies in different parts of the cusp region. These facts further confirm the cusp's potent connectivity with both the solar wind and the equatorial radiation belt in the presence of a significant IMF $B_{y}$ component.

Convection Electric Field: The role of the convection electric field can also be predicted. Because of the dawndusk orientation of the electric field, particles can be accelerated when they travel duskward. Thus, the conservation of 
the magnetic momentum $\mu$ requires that the $|B|$ of the magnetic mirror point should also increase, which consequently enlarges the value of CTL. So, during the interval of enhanced convection, the cusp's capability of trapping particles in the duskside sector is weakened, and the particles are more inclined to escape into the duskside peak. Less energetic particles should be more sensitive to this effect, because of the relatively larger percentage of change in their energy.

We use the test particle approach again in T96 along with the DICM electric field model (Papitashvili and Rich, 2002) to examine the conclusion. The trajectory of a $50 \mathrm{keV}$ proton initially with a $90^{\circ}$ pitch angle is calculated and shown in Fig. $6 \mathrm{~b}$ as the red line. Instead of following a closed orbit, the particle escapes from the "cusp minimum plane" in the duskside sector, and joins up with the solar wind (because of the existence of negative IMF $B_{y}$ ).

In brief, strong convection would favor cusp particle leakage from the duskside sector, connecting the leakage particles to the solar wind in the case of negative IMF $B_{y}$ or merging them to the dipole radiation belt during positive or zero IMF $B_{y}$.

Birkeland current system: If we study the $90^{\circ}$ test particle's "closed orbit" carefully enough, we will discover that the orbit is not perfectly closed, which implies that the first adiabatic invariant $\mu$ is not exactly conserved during a drift circle. The nonadiabatic behavior, first discovered by Kremser et al. (1995) and subsequently described by Zong et al. (2005) without a satisfactory explanation, is identified to be caused by the Birkeland current system in the T96 model. The current which flows in relatively thin sheets can produce a significant $\mathrm{B}$ variation over a small distance, and thus will remotely modifiy the geometry of cusp.

Although the variation of $\mu$ is not significant, test particles can still shift their "cusp-shells" and even move away from the "cusp minimum plane" after drifting for a relatively long time. Figure $6 \mathrm{c}$ presents the effect of Birkeland current system. The $100 \mathrm{keV}$ proton initially with a $90^{\circ}$ pitch angle drifts around the cusp for six times, with $\mu$ decreasing by a total of $20 \%$, and finally becomes detrapped to the equatorial trapping region. The non-adiabatic motion, as an effect of Birkeland current system, can probably decrease the steadiness of the "cusp radiation belt", and also increase the possibility of particle exchange between the cusp and other regions.

In order to verify our conclusion, we perform exactly the same test particle simulation in a T96 model with the Birkeland term being removed. The resulting first adiabatic invariant $\mu$ never changes, and the $90^{\circ}$ proton remains trapped forever (not shown here).

It is worth noting that the goal of our test particle simulations is to present the coexistence of the bounce and the drift motion of energetic particles in the cusp region, to test the particle behavior under different conditions, as can be predicted by the CTL spatial distribution, and to verify the connectivity of the cusp with distinct regions for different cusp sectors. We do not expect a real particle to experience the whole orbit, but instead, the particle will follow a certain part of the orbit and then switch to another one because of the time dependence of CTL, which can also change the extent of the stably-trapped region of the "cusp minimum plane", driving some of the originally-trapped particles away from the cusp region. Both the "sticky" nature for IMF directions (Zhou et al., 1997; Cargill et al., 2005) and the strong fluctuations with a $d B / B$ of nearly 1 within a few minutes (e.g. Sheldon et al., 1998; Zhang et al., 2005) are able to modify the CTL distribution and hence activate the particle exchange process between the equator, the cusp, and the solar wind.

Based on the statistical study of Lavraud et al. (2004) and Lavraud and Cargill (2005) that the magnetic field in the cusp is even weaker than the prediction of T96, it should also be noted that the "cusp trapping limit" in our calculation are probably overestimated, and the particle trapping capability of the cusp region may be stronger. To obtain a better estimation of the cusp's role on particle trapping and exchanging, a more accurate magnetospheric magnetic model is strongly required and highly desirable.

\section{Discussion and summary}

We have displayed the T96 topology of the off-equatorial field minimum region, or "cusp radiation belt", and thus have studied its effect on the behavior of energetic particles. Those particles with near $90^{\circ}$ pitch angles appear to be trapped by the field minimum and drift around the cusp via closed orbits if no temporal effects are considered. Numerical simulations that were performed demonstrate the existence of near $90^{\circ}$ particle trapping in the cusp, which was also well observed by several satellites (Antonova et al., 1999; Fritz et al., 2000; Zong et al., 2004).

It is of importance to notice the coexistence of open field lines and closed field lines within the same drift orbit in the cusp. Particles may be temporarily trapped in the cusp region, drift for some time and escape to different places. It has been shown that the special magnetic topology in the cusp region can provide a window for particle exchange between the solar wind, the cusp, and the radiation belt, suggesting a possible way of equatorial particle leakage or solar wind particle injection into the magnetosphere.

The spatial structure of the "cusp minimum plane" under different conditions can further give us some interesting information on the cusp particle motion dependency on the tilt angle of the Earth's dipole axis, the components of the IMF, the convection electric field and the Birkeland current system. In different positions and under different conditions, the capability of trapping particles can be distinctly different, thus providing us with clues on determining when and where the particle exchange process would most probably happen. 
A set of test particle simulations in the T96 model are presented to prove these results. To make a better prediction, a more accurate magnetic model in the cusp region is required.

Acknowledgements. We are grateful to N. A. Tsyganenko, H. Zhang, P. Wu and X. G. Zhang for their helpful suggestions and discussions in this paper. The work was initiated during a visit by X.-Z. Zhou to Boston University in Fall/Winter 2004, supported by NASA grant NAG5-10108. This work is also supported by NSFC project 40528005, 40390152 and the International Collaboration Research Team Program of the Chinese Academy of Sciences.

Topical Editor I. A. Daglis thanks I. Sandahl and another referee for their help in evaluating this paper.

\section{References}

Antonova, A. E. and Shabansky, V. P.: Structure of the geomagnetic field at great distance from the Earth, Geomagn. Aeron., 8, 801811, 1968.

Antonova, A. E., Gubar, Y. I., and Kropotkim, A. P.: Energetic particle population in the high-latitude geomagnetosphere, Phys. Chem. Earth, 25, 47-50, 1999.

Cargill, P. J., Lavraud, B., Owen, C. J., Grison, B., Dunlop, M. W., Cornilleau-Wehrlin, N., Escoubet, C. P., Paschmann, G., Phan, T. D., Rezeau, L., Bogdanova, Y., and Nykyri, K.: Cluster at the Magnetospheric Cusps, Space Sci. Rev., 118, 321-366, doi: 10.1007/s11214-005-3835-0, 2005.

Delcourt, D. C. and Sauvaud, J.-A.: Recirculation of plasma sheet particles into the high-latitude boundary layer, J. Geophys. Res., 103, 26 521-26 532, 1998.

Delcourt, D. C. and Sauvaud, J.-A.: Populating of Cusp and Boundary Layers by energetic (hundreds of $\mathrm{keV}$ ) equatorial particles, $\mathrm{J}$. Geophys. Res., 104, 22 635-22 648, 1999.

Fritz, T. A., Chen, J., and Sheldon, R. B.: The Role of the Cusp as A Source for Magnetospheric Particles: A New Paradigm?, Adv. Space Res., 25, 1445-1457, 2000.

Fuselier, S. A., Anderson, B. J., and Onsager, T. G.: Particle signatures of magnetic topology at the magnetopause: AMPTE/CCE observations, J. Geophys. Res., 100, 11 805-11 821, 1995.

Kirpichev, I., Fedorov, A., Grigoriev, A., Budnik, E., and Dubinin, E.: Quasi-trapping of Charged Particles in the Region of a Local Magnetic Field Minimum in the Outer Cusp, Cosmic Research, 37, 638-643, 1999.

Kremser, G., Woch, J., Mursula, K., Tanskanen, P., Wilken, B., and Lundin, R.: Origin of energetic ions in the polar cusp inferred from ion composition measurements by the Viking satellite, Ann. Geophys., 13, 595-607, 1995, http://www.ann-geophys.net/13/595/1995/.

Lavraud, B. and Cargill, P. J.: Cluster reveals the magnetospheric cusps, Astronomy and Geophysics, 46, 1.32-1.35, 2005.

Lavraud, B., Fedorov, A., Budnik, E., Grigoriev, A., Cargill, P. J., Dunlop, M. W., Rème, H., Dandouras, I., and Balogh, A.: Cluster survey of the high-altitude cusp properties: a three-year stastical study, Ann. Geophys., 22, 3009-3019, 2004,

http://www.ann-geophys.net/22/3009/2004/.
Merka, J., Safránková, J., and Nemecek, Z.: Cusp-like plasma in high altitudes: a statistical study of the width and location of the cusp from Magion-4, Ann. Geophys., 20, 311-320, 2002, http://www.ann-geophys.net/20/311/2002/.

Newell, P. T. and Meng, C. I.: Cusp Width and $B_{z}$ : Observations and a Conceptual Model, J. Geophys. Res., 92, 13 673-13678, 1987.

Papitashvili, V. O. and Rich, F. J.: High-latitude ionospheric convection models derived from Defense Meteorological Satellite Program ion drift observations and parameterized by the interplanetary magnetic field strength and direction, J. Geophys. Res., 107, doi:10.1029/2001JA000264, 2002.

Pugacheva, G., Gusev, A. A., Jayanthi, U. B., Schuch, N. J., and Spjeldvik, W. N.: Seasonal polar cap radiation zones in the dayside magnetosphere, Earth Planets Space, 56, e17-e20, 2004.

Pugacheva, G., Gusev, A. A., Jayanthi, U. B., Schuch, N. J., and Spjeldvik, W. N.: Cusp region radiation belts in the dayside magnetosphere, J. Atmos. Solar-Terr. Phys., 67, 479-487, 2005.

Shabansky, V. P.: Some processes in the magnetosphere, Space Sci. Rev., 12, 299-418, 1971.

Sheldon, R. B., Spence, H. E., Sullivan, J. D., Fritz, T. A., and Chen, J.: The discovery of trapped energetic electrons in the outer cusp, Geophys. Res. Lett., 25, 1825-1828, 1998.

Tsyganenko, N. A. and Russell, C. T.: Magnetic signatures of the distant polar cusps: Observations by Polar and quantitative modeling, J. Geophys. Res., 104, 24 939-24 955, 1999.

Tsyganenko, N. A. and Stern, D. P.: Modeling the global magnetic field of the large-scale Birkeland current system, J. Geophys. Res., 101, 27 187-27 198, 1996.

Zhang, H., Fritz, T. A., Zong, Q. G., and Daly, P. W.: Stagnant Exterior Cusp Region as Viewed by Energetic Electrons and Ions: A Statistical Study Using CLUSTER Research with Adaptive Particle Imaging Detectors (RAPID) Data, J. Geophys. Res., 110, A05211, doi:10.1029/2004JA010562, 2005.

Zhou, X.-W., Russell, C. T., Le, G., and Tsyganenko, N.: Comparison of observed and model magnetic fields at high altitudes above the polar cap: Polar initial results, Geophys. Res. Lett., 24, 1451-1454, 1997.

Zhou, X. W., Russell, C. T., Le, G., Fuselier, S. A., and Scudder, J. D.: Solar wind control of the cusp at high altitude, J. Geophys. Res., 106, 245-251, 2000.

Zong, Q.-G., Fritz, T. A., Zhang, H., Korth, A., Daly, P. W., Dunlop, M. W., Glassmeier, K.-H., Reme, H., and Balogh, A.: Triple cusps observed by Cluster - Temporal or spatial effect?, Geophys. Res. Lett., 31, doi:10.1029/2003GL019128, 2004.

Zong, Q. G., Fritz, T. A., Korth, A., Daly, P. W., Dunlop, M., Balogh, A., Fennell, J. F., Sullivan, J. D., Friedel, R. W. H., and Reme, H.: Energetic Electrons as a Field Line Topology Tracer in the High Latitude Boundary/CUSP Region: Cluster Rapid Observations, Surveys in Geophysics, 26, 215-240, doi:10.1007/s10712-005-1879-z, 2005. 